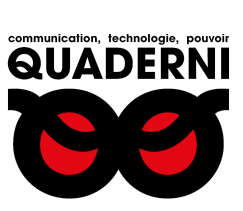

\title{
Quaderni
}

Communication, technologies, pouvoir

96 | Printemps 2018

Smart city : "fiction" et innovation stratégique

\section{Fake news et post-vérité. De l'extension de la propagande au Royaume-Uni, aux États-Unis et en France}

Patrick Troude-Chastenet

\section{(2) OpenEdition \\ Journals \\ Édition électronique \\ URL : https://journals.openedition.org/quaderni/1180 \\ DOI : 10.4000/quaderni. 1180 \\ ISSN : 2105-2956 \\ Éditeur \\ Les éditions de la Maison des sciences de l'Homme}

Édition imprimée

Date de publication : 15 mai 2018

Pagination : 87-101

Référence électronique

Patrick Troude-Chastenet, « Fake news et post-vérité. De l'extension de la propagande au RoyaumeUni, aux États-Unis et en France », Quaderni [En ligne], 96 | Printemps 2018, mis en ligne le 15 mai 2020, consulté le 28 septembre 2021. URL : http://journals.openedition.org/quaderni/1180 ; DOI : https://doi.org/10.4000/quaderni. 1180 


\section{Politique}

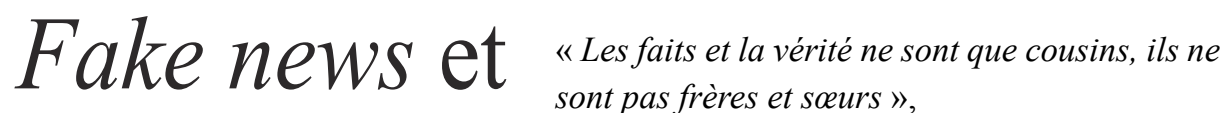

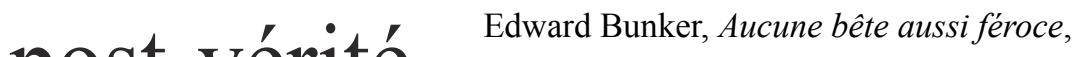
post-Vérité. Rivages Noir, 2016.

De l'extension de la propagande au Royaume-Uni, aux États-Unis et en France

\section{Patrick Troude-Chastenet}

Professeur de science politique Université de Bordeaux, Centre Montesquieu de Recherche Politique
"La liberté d'opinion est une farce si l'information sur les faits n'est pas garantie et si ce ne sont pas les faits eux-mêmes qui font l'objet du débat ",

Hannah Arendt, "Vérité et politique », in $L a$ Crise de la culture : huit exercices de pensée politique,

Gallimard, coll. Folio Essais, Paris, 1989 (1967).

«Il n'a pas prononcé des contre-vérités, Chuck, il a proposé des faits alternatifs » répond, le sourire un rien crispé, Kellyanne Conway, conseillère à la Maison Blanche, au célèbre animateur de l'émission Meet the Press qui lui demande pourquoi le porte-parole ${ }^{1} \mathrm{du} 45^{\mathrm{e}}$ président des États-Unis avait affirmé la veille, à tort, que sa cérémonie d'investiture avait attiré plus de public que celles de Barak Obama. Et Chuck Todd, directeur du département politique de $N B C N e w s$, de manquer de s'étrangler devant cette tentative de théorisation du dernier avatar de la nouvelle ère de la communication-propagande inaugurée bien avant la double victoire de Trump et du Brexit : « Des faits alternatifs? Mais ce ne sont pas des faits, ce sont des mensonges. » La veille encore, le 21 janvier 2017, Donald Trump prétendait également que le soleil avait remplacé la pluie dès qu'il avait commencé son discours d'inauguration alors que les caméras de télévisions montraient, parmi les parapluies, l'ancien président George W. Bush se couvrant la tête d'un poncho en plastique transparent. Que nous apprend sur nos démocraties ce prétendu droit à être « en désaccord avec 
les faits ${ }^{2} »$, dont se réclament nombre de leaders «populistes»?

Commençons donc par ne pas écarter tous les faits et par définir le sens des mots. Au sens strict, les fake news ne sont pas seulement des fausses nouvelles mais des informations volontairement truquées, falsifiées et trompeuses ${ }^{3}$. L'expression devrait donc désigner uniquement le faux article de presse, délibérément inexact et publié sur un faux site d'actualités, destiné à abuser ou à manipuler le lecteur. L'un des plus célèbres du genre, le faux communiqué du Vatican affirmant que le pape François ${ }^{4}$ soutenait la candidature de Donald Trump 5 , illustre d'ailleurs la porosité entre les trois catégories d'émetteurs de $f a k$ news rencontrées sur la Toile : les satire website comme The Onion.com aux États-Unis ou Le Gorafi, en France, dont l'objectif est l'humour ; les sites conspirationnistes qui prennent parfois la forme de clickbait site, autrement dit de pièges à clics, permettant de générer des revenus publicitaires et enfin, les Fake News Website qui diffusent des hoax, des rumeurs, des canulars en tout genre. En effet, le faux communiqué du pape diffusé par le site conspirationniste d'extrême droite Ending the Fed, largement relayé sur les réseaux sociaux au point d'être l'information la plus virale de la campagne présidentielle, provenait à l'origine de WTO5 News, un site qui avertissait pourtant sur sa page d'accueil que la plupart de ses articles relevait de l'imagination et de la satire. Dans le même sens, c'est à partir d'un article du site parodique Le Gorafi attribuant au candidat Emmanuel Macron des propos peu amènes à l'égard des défavorisés ${ }^{6}$, partagé plus de 600000 fois avant le second tour de l'élection présidentielle, que plusieurs sites de droite et d'extrême droite ont sciemment accrédité l'idée selon laquelle il s'essuyait les mains dès qu'elles entraient en contact avec celles des pauvres. Il faudrait également pouvoir distinguer les motivations mercantiles des objectifs strictement politiques, la diffusion par le haut (un leader politique) de la diffusion par le bas ( $«$ journalisme citoyen », activistes bénévoles).

Outre-Atlantique, en dépit de la logique et du bon sens, des millions d'Américains ont cru que le souverain pontife soutenait le candidat républicain, comme ils ont bien voulu croire que sa rivale, Hilary Clinton, avait vendu des armes à Daesh et que cette organisation appelait les musulmans vivant aux États-Unis à voter démocrate $^{7}$. Au sens large, et la banalisation dans notre langage quotidien du recours au mot anglais est déjà un indicateur de la réalité qu'il désigne, le terme de fake news peut déborder des limites de la contrefaçon et s'appliquer à toute information délibérément mensongère, indépendamment de la nature du media utilisé. Quoiqu'il en soit, les fake news ont partie liée avec la tromperie et la manipulation, ce qui les inclut - en tant que simple technique - dans un phénomène beaucoup plus vaste et par ailleurs millénaire : la propagande $^{8}$, déjà utilisée par le tyran Pisistrate, parmi d'autres, dès le $\mathrm{VI}^{\mathrm{e}}$ siècle avant Jésus-Christ ${ }^{9}$.

La combinaison de ces informations mensongères et trompeuses (fake news), en très forte augmentation depuis 2015, et de ces prétendus faits alternatifs (alternative facts) nous a-t-elle fait basculer imperceptiblement dans un nouveau régime de vérité et de mensonge connu désormais sous le nom de post-vérité (post-truth) ? Cette question en appelle beaucoup d'autres. Ce phéno- 
mène est-il aussi nouveau qu'on le dit ? S'il n'est pas totalement inédit, peut-on se contenter pour autant d'y voir la permanence de la bonne vieille propagande, et de la poursuite du bidonnage sous d'autres moyens, derrière le masque informatisé des nouvelles formes d'intox ${ }^{10}$ ? S'il $\mathrm{y}$ a du nouveau, où est-il et en quoi consiste-t-il exactement? Faut-il l'attribuer à l'élargissement de l'espace public provoqué par la révolution numérique? Quel rôle jouent les réseaux sociaux dans cette affaire ? Existe-t-il en la matière un tropisme américain, ou pour le moins anglo-saxon (Donald Trump et ses émules britanniques), qui devrait donc, si ce n'est déjà fait, gagner lentement mais inexorablement 1 'Europe entière ? Ce phénomène est-il à considérer comme une simple dérive du système médiatique, due pour l'essentiel à l'outil informatique, et accessoirement à la force de la propagande russe en ligne, ou traduit-il, plus profondément, un nouveau rapport démocratique à la vérité et au mensonge dont chacun se fait et se fera tour à tour l'expert, le complice ou la victime ? Ce pluralisme des vérités n'est-il pas non plus à interpréter à l'aune de la défiance généralisée envers les institutions (État, école, média) et leurs élites ? La post-vérité et les discours nationaux-populistes ont-ils été engendrés, des deux côtés de l'Atlantique, par une décennie de sirènes post-nationales et d'asepsie politiquement correcte ${ }^{11}$ ? Tenter d'esquisser une réponse à cette dernière question suppose au préalable de ne pas esquiver les précédentes.

\section{Les faits ne fonctionnent pas !}

Si selfie fut élu mot de l'année en 2013, post-truth lui succéda en 2016. Selon l'Oxford Dictionnary, ce concept sert à désigner les « circonstances dans lesquelles les faits objectifs ont moins d'influence sur l'opinion publique que les appels à l'émotion ou aux opinions personnelles ». Commentant le succès du Brexit, l'un de ses partisans expliquait sa victoire par le fait que les électeurs préféraient que l'on s'adresse à leurs tripes plutôt qu'à leur raison ${ }^{12}$. Facts don 't work ! résumait le milliardaire Aaron Banks, cofondateur de la plateforme Leave.EU et principal soutien financier de l'Ukip. Les responsables de la communication des Brexiters se sont résolument inscrits dans la filiation trumpiste en matière d'informations tronquées, fausses ou purement fantaisistes ${ }^{13}$, affirmant notamment que cinq millions d'étrangers supplémentaires en provenance des Balkans et de la Turquie seraient autorisés à franchir le Channel d'ici 2030, que l'appartenance à l'UE coûtait au Royaume-Uni pas moins de 350 millions de $£$ par semaine privant d'autant les caisses du National Health Service (le système de santé publique), dénonçant en une du Daily Mail « le complot destiné à faire entrer 1,5 million de Turcs en Grande-Bretagne » ou, affiches à l'appui, les milliers de réfugiés et migrants supposés fouler le sol britannique alors qu'en réalité ils étaient massés à la frontière slovèno-croate ${ }^{14}$. Pour chacun des thèmes utilisés par les Brexiters durant cette campagne référendaire, il fut loisible de constater qu'aucun démenti, qu'aucune statistique produite par aucun organisme officiel ${ }^{15}$, qu'aucune parole d'expert réputé neutre n'était audible pour la partie du public qui avait envie de croire à ce qui lui semblait crédible. D'autant que le pire était à craindre à en croire les deux camps. L'argumentaire des partisans du retrait du Royaume-Uni de l'UE se polarisa sur l'immigration, avec des accents ouvertement xénophobes, alors que celui des 
partisans du maintien se situa résolument sur le registre économique et financier sans doute trop abstrait pour rassurer la majorité de l'électorat. Cela dit, ces derniers se placèrent également sur le terrain des émotions en jouant sur la peur d'une partie de l'électorat de voir s'effondrer le cours de la livre sterling et le prix de l'immobilier, augmenter le prix des transports aériens et des séjours touristiques à l'étranger. Surtout, « le Projet Panique ${ }^{16}$ », insistait sur la perte de milliers d'emplois qui résulterait immanquablement d'une sortie de l'UE. Peur de l'invasion versus peur de l'inconnu' ${ }^{17}$. Gardons-nous bien de la moquerie car, comme nous le rappelle le fabuliste préféré des Français, « Chacun croit aisément ce qu'il craint et ce qu'il désire ${ }^{18}$. » Difficile d'évaluer le poids des fake news dans la victoire des pro-Brexit dont plusieurs ténors se réclamèrent ouvertement des méthodes de Donald Trump pour emporter les primaires.

La parenté revendiquée semble légitime mais il convient de remonter un peu plus loin dans le temps car le $45^{\mathrm{e}}$ président des États-Unis n'est pas l'inventeur d'un genre qui commença en 1999 sur le mode de la satire avec le Daily Show et qui prit son sens et sa forme actuelle en 2004 pour exploser littéralement en $2016^{19}$. Avant ces deux dates nous avions affaire à de la parodie ou à de la désinformation classique dans la mesure où elle ne relevait pas directement de la contrefaçon. Néanmoins, on s'aperçoit que quelque chose vient de changer dans le régime de la vérité. Des dirigeants supposés responsables d'une grande nation démocratique s'affranchissent d'autant plus allégrement du lien avec la réalité empirique qu'ils fabriquent le récit que leur public attend d'eux. Développant les thèses sur le ration- nement des soins défendues en 1994 dans The New Republic contre le Hillarycare par la très conservatrice Elizabeth McCaughey ${ }^{20}$, la républicaine Sarah Palin, candidate à la vice-présidence des ÉtatsUnis en 2008, répandit l'idée selon laquelle la réforme du système de santé prévoyait des death panels ${ }^{21}$ obtenant ainsi le prix du «mensonge politique de l'année » décerné par le site internet de vérification des faits Politifact en 2009. Selon la colistière de John McCain en effet, les démocrates avaient l'intention de créer des comités contrôlés par le gouvernement qui décideraient si oui ou non les personnes âgées ou handicapées mériteraient de recevoir des soins. Selon le site PolitiFact, environ $30 \%$ de la population a cru sincèrement que les death panels faisaient partie intégrante de la nouvelle loi sur la protection des malades ${ }^{22}$. En 2011, les membres du Parti républicain dénoncèrent une prétendue recrudescence des fraudes électorales aux États-Unis alors que les chiffres indiquaient le contraire ${ }^{23}$. Lors de la campagne présidentielle de 2012, Mitt Romney déclara que 47\% des foyers américains percevaient une aide de la part du gouvernement d'une manière ou d'une autre ce qui les inciterait à voter pour Barack Obama ${ }^{24}$. Mais c'est seulement à partir de 2015 qu'il peut éventuellement être question d'un basculement dans l'ère de la post-vérité ${ }^{25}$. En décembre de la même année, Politifact révéla que pas moins de $76 \%$ des déclarations de Donald Trump durant la campagne présidentielle étaient «principalement fausses, fausses ou de pures inventions ${ }^{26}$. » En 2016, ce site de fact-checking décerna «le prix du mensonge de l'année » aux fake news dans leur ensemble, dans l'acception stricte du terme ${ }^{27}$. Même si personne ne conteste le caractère ancestral de la circulation des rumeurs et 
des pratiques de désinformation, - on se souvient tous du « message important» à transférer à tous nos amis -, le caractère industriel de la fabrique de nouvelles contrefaites et la viralité de leur diffusion n'a plus grand-chose à voir avec la bonne vieille chaîne de lettres via la Poste ou le courriel. Analysant l'impact des fake news sur l'élection présidentielle, le site BuzzFeed a révélé que durant les trois derniers mois de la campagne des informations délibérément fausses avaient suscité plus de partages, de réactions et de commentaires sur Facebook que celles de CNN, du New York Times et du Washington Post ${ }^{28}$. Outre la vente d'armes à Daesh et le financement de l'organisation islamiste, Hillary Clinton fut notamment accusée de diriger un réseau de pédophiles (Pizzagate) ${ }^{29}$, d'avoir fait maquiller en suicide le meurtre de l'agent du FBI qui enquêtait sur l'usage frauduleux de sa messagerie privée ou encore d'avoir encouragé le parti démocrate à imposer la loi islamique en Floride. Ces informations, résolument inexactes et malveillantes, émanaient en apparence de source journalistique et furent partagées des millions de fois sur les réseaux sociaux durant la campagne présidentielle. Au lendemain de sa victoire, c'est Trump lui-même qui fut victime de fake news puisque circula sur les réseaux la rumeur selon laquelle plusieurs milliers de ses supporters l'acclamaient à Manhattan aux cris de : "Nous haïssons les musulmans, nous haïssons les Noirs, faisons que notre pays redevienne grand ${ }^{30}$. »

\section{Une spécialité américaine?}

Les Américains sont-ils un peuple plus crédule que les autres comme l'on se plaît à le penser de l'autre côté de l'Atlantique ? Le sont-ils plus, par exemple, que les Russes, grands amateurs de théories conspirationnistes, dont on ne peut pas dire que les dirigeants successifs, de l'ère tsariste au régime de Poutine en passant par la dictature stalinienne, aient eu le souci d'éduquer dans le culte de la vérité historique ? Il est sans doute moins utile de convoquer « l'âme des peuples » chère à Herder, Tocqueville et André Siegfried que de s'en tenir à des considérations strictement empiriques. Les Américains sont surtout plus connectés mais ils sont aussi plus nombreux à admettre s'informer principalement en ligne (70\%), y compris sur les réseaux sociaux dont Facebook et Twitter $^{31}$ (51\%) contre à peine 17\% en France en $2016^{32}$. Ils sont par ailleurs, pour l'immense majorité d'entre eux, convaincus des mérites du libéralisme sous toutes ses formes. Toutes les informations, comme toutes les idées y compris les plus fantaisistes ou délirantes, doivent pouvoir circuler librement. Cette conviction a fait la fortune de quelques jeunes travailleurs du clic en Macédoine et de plusieurs webmestres aux États-Unis ${ }^{33}$. Pas moins de 150 noms de domaines ont été identifiés dans la ville de Vélès, désormais célèbre pour ses usines à fake news et les titres racoleurs de ses faux sites : «Ce qui est caché dans la jambe droite du pantalon de Hillary Clinton lui coûtera l'élection présidentielle » (Usa News Flash), "L'ancien chasseur de prime de Clinton avoue avoir tué des gens pour de l'argent » (Usa Daily Politics). Entourés de bannières publicitaires, ces prétendues révélations relayées ensuite sur les réseaux sociaux permirent à de jeunes Macédoniens d'obtenir dix fois le salaire moyen de leur pays et à des webmestres californiens qui versaient 3000 \$ par mois à Facebook pour promouvoir leurs articles pro-Trump de multiplier par dix en moyenne leur 
mise $^{34}$. L'industrie des fake news n'a donc pas fini de prospérer tant elle est consubstantielle à l'économie néolibérale. D'autant que le numérique permet de « radicaliser l'idée libérale d'un espace public appréhendé comme un vaste marché des idées ${ }^{35}$ où la vérité est la simple résultante d'une mise en concurrence des informations ${ }^{36}$. » $\mathrm{Au}$ nom des valeurs libérales défendues par la Constitution, les thèses créationnistes, racistes ou climatosceptiques ont droit de cité au même titre que la théorie darwinienne de l'évolution, les idées antiracistes ou l'idéologie écologiste.

\section{Un facteur Trump?}

Existe-il un facteur Trump venant accréditer la thèse d'un basculement dans l'ère de la postvérité $^{37}$ ? L'équation personnelle du $45^{\mathrm{e}}$ président n'est sans doute pas à négliger. Le New York Times publia une liste de ses déclarations faisant apparaître que lors de ses quarante premiers jours à la Maison-Blanche seuls quatre n'avaient pas été entachés de «mensonge » ou de " manque de franchise » (falsehood $)^{38}$. On pourrait bien sûr évoquer les précédents de Ronald Reagan niant toute transaction avec l'Iran pour la libération des otages ou encore les mensonges de l'administration Bush à propos de la détention par l'Irak d'armes de destruction massive mais, dans les deux cas, ces mensonges d'État ne sont pas imputables à l'idiosyncrasie des acteurs mais à des logiques systémiques. A contrario tout porte à croire qu'avec Donald Trump l'exception soit devenue la règle, grâce et avec la complicité de son électorat qui se pense comme seule incarnation de l'Amérique éternelle trahie par des élites mondialisées, multiculturelles et engluées dans le politiquement correct et qui n'attend pas de lui la description policée d'une vérité complexe et nuancée mais un discours tellement simplificateur qu'il en devient manichéen, qui s'adresse à ses émotions plutôt qu'à sa raison et qui ne répugne pas, à l'occasion, à emprunter au registre du « savoir stigmatisé » des théories complotistes ${ }^{39}$. Loin de lui nuire, les mensonges lui profitent tant le propagandé est complice du propagandiste, comme l'avait noté en son temps Jacques Ellul $^{40}$. Lors d'une conférence à Harvard, l'un de ses proches conseillers s'en prit aux journalistes non pour l'avoir critiqué mais pour lui avoir fait crédit : «Vous avez pris trop littéralement ce que disait Donald Trump. Mais pas les Américains. Eux ont compris que, parfois, quand vous parlez avec les gens... vous allez dire des choses, et quelquefois, il vous manque des choses pour les étayer ${ }^{41}$. " Edward Bernays, le père spirituel de tous les spin doctors, expliquait volontiers que puisque ses contemporains ne se passionnaient pas pour la politique il fallait piquer leur curiosité en s'adressant à leurs intérêts particuliers avant de conclure : «La propagande est utile au politicien si, et seulement si, le public, consciemment ou non, a envie d'entendre ce qu'il a à dire ${ }^{42}$. » On peut parfaitement admettre l'hypothèse que, durant la campagne présidentielle, ni Trump ni ses supporters ne croyaient à toutes les bullshit sortant de sa bouche mais visiblement il prenait autant de plaisir à les proférer qu'eux à les entendre ${ }^{43}$. Dans Sale histoire, Éric Ambler met en scène un personnage à qui son père avait donné ce conseil en héritage : «Ne dis jamais de mensonge si tu peux t'en tirer par du baratin ${ }^{44}$. » De ce point de vue, Trump est peut-être un menteur mais il est surtout un bullshiter, un baratineur. Si la différence est parfois subtile entre toutes les nuances du faux, si la frontière entre la vérité 
et l'erreur est souvent poreuse, Trump a montré qu'il n'avait pour la rectitude et l'aridité des faits que mépris ou indifférence. De façon symptomatique, Whatever (peu importe) et Who cares ? (« qu'est-ce que ça peut faire ?») sont deux de ses expressions favorites pour échapper à ses contradicteurs. «Désormais, résume à son propos Ran Halévi, il ne s'agit plus, comme autrefois, de trahir la vérité, ou de la déformer, ou de la contourner, ou encore de la recréer rétrospectivement, mais de l'ignorer tout simplement, parce que trop complexe, trop encombrante, rébarbative, sans appétence, voire suspecte ${ }^{45}$. »

\section{Vieille propagande et nouvelle intox ?}

Si la campagne présidentielle française fut également polluée par le phénomène des fake news, elle confirma aussi la persistance de la propagande traditionnelle à l'ère numérique. Finalement vainqueur, le candidat Macron fut successivement accusé d'être financé par l'Arabie Saoudite, de vouloir instaurer la charia à Mayotte, de mener une double vie avec un responsable de l'audiovisuel public, d'avoir menti sur son patrimoine, de vouloir faire payer un loyer aux propriétaires et enfin, lors du débat télévisé précédant le second tour, de posséder un compte offshore aux Bahamas. Rumeur émanant du célèbre forum anonyme anglophone 4chan reprise en direct par Marine Le $\mathrm{Pen}^{46}$. Plusieurs semaines auparavant, les sites Riposte Laïque et Boulevard Voltaire s'en étaient donnés à cœur joie sur le thème du " grand mufti de Bordeaux Ali Juppé » et de son "projet pharaonique » de construction de mosquée sur les bords de la Garonne, financée par l'Azerbaïdjan et le Qatar. À l'occasion des primaires de la droite, cette campagne de l'extrême droite profita largement au camp filloniste qui s'embourba à son tour dans une affaire, à l'ancienne, le Penelopegate, révélée par un media on ne peut plus traditionnel, Le Canard Enchaîné, une institution du genre. Puisque nouveauté il y a, elle réside avant tout dans le caractère viral de ces informations, vraies ou fausses, relayées sur les réseaux sociaux et les forums de discussion.

Même si cette thèse est contestée, la circulation de l'information sur la Toile est soumise à ce que l'on appelle une bulle de filtres ou bulles filtrantes dont l'existence même contredit la vision habermassienne d'une démocratie numérique fondée sur un accès égal pour tous à la connaissance et à une capacité partagée de discuter publiquement et rationnellement de sujet d'intérêt général ${ }^{47}$. Alors qu'apparemment la masse d'informations n'a jamais été aussi vaste et aussi rapidement disponible, l'information non filtrée par les algorithmes deviendrait une denrée d'autant plus rare que nos choix personnels antérieurs sur la Toile nous isoleraient en raison d'une logique cumulative caractéristique du cercle vicieux. $\mathrm{Si}$ on leur prête parfois trop de pouvoir les mécanismes de sélection algorithmique opèrent quotidiennement, à notre insu, pendant et hors des périodes électorales. Un utilisateur de Google souhaitant s'informer sur l'état de la Turquie en juillet 2016, en fonction de son historique de recherche et donc de son profil, verra s'afficher en première page des sites touristiques, des hôtels et des vols bon marché alors qu'un autre sera dirigé sur les dernières nouvelles de la répression dans ce pays. Mais les algorithmes ont également un lien direct avec les fake news dans la mesure où ces dernières sont sans intérêt pour l'émetteur 
s'il n'est pas en mesure de les partager à grande échelle via notamment ce que l'on appelle des bots, des programmes capables d'automatiser la diffusion de contenus, de créer artificiellement une vague de popularité, une tendance qui leur permettra d'entrer dans le flux de mises à jour de Facebook $^{48}$. Pour nous atteindre, il suffit qu'un seul de nos amis l'ait validée en l'intégrant dans son fil d'actualités alors que la viralité de cette information repose sur un distributeur automatique installé dans un centre commercial à Moscou ou sur des « travailleurs du clic » basés en Macédoine ou en Thaïlande, payés à la tâche ${ }^{49}$ qui moyennant un dollar environ permettent d'acheter cent faux likes pour un message posté sur les réseaux sociaux et cent nouveaux abonnés. En outre, l'effet bulle de filtres peut se combiner avec l'effet chambre d'écho qui accentue le biais de confirmation. Mes amis pensent comme moi sinon ils ne seraient pas mes amis. L'information partagée sur mon fil d'actualités renforce mes propres préjugés et prédispositions en m'exposant à une propagande univoque. Néanmoins, il ne faut pas se contenter d'une explication par le support technique, en l'occurrence l'internet, mais replacer cet outil dans le cadre d'une économie de l'attention elle-même tributaire du technocapitalisme néolibéral. «Tout comme il existe des agents économiques qui produisent des fake news ayant des effets politiques, il existe des agents politiques qui produisent des fake news ayant des effets politiques et économiques ${ }^{50}$. » Pour fonctionner, le modèle économique des fake news a besoin des grandes plateformes d'intermédiation telle que les moteurs de recherche (Google), les réseaux sociaux (Facebook, Twitter, etc.) et les réseaux de publicité tels que Google Ads. Pour une large part, les GAFA sont donc sinon politiquement complices mais pour le moins économiquement solidaires de l'industrie des fake news car, en définitive, ils en sont les principaux bénéficiaires. L'espace public numérique est dominé par «des dynamiques de circulation de l'information favorisant les contenus sensationnalistes - factuellement établis ou non - qui attirent les clics ${ }^{51}$. »

\section{Conclusion}

S'il est peut-être encore trop tôt pour accréditer la thèse d'un basculement dans l'ère de la postvérité, la prégnance des fake news dans notre quotidien, depuis 2016, interdit de parler d'une simple dérive du système médiatique imputable à la seule révolution numérique. Ce phénomène est inséparable de la démocratie d'opinion et de la conception libérale de l'information voulant que la vérité résulte naturellement de la mise en concurrence sur le marché des idées de tous les contenus, falsifiés ou authentiques. Faisant de nous tous des citoyens compétents en matière politique, le régime démocratique aiguise notre sens critique à l'égard des vérités officielles sans pour autant diminuer notre crédulité. Le modèle démocratique postule l'existence de citoyens actifs, rationnels, instruits et informés mais que signifie exactement s'informer alors que pour le faire sinon objectivement mais du moins équitablement il faudrait pouvoir disposer à la fois de beaucoup de temps et de discernement, deux ressources devenues rares. Que veut dire s'informer dans un pays et à une époque où il en coûte un euro pour acheter un livre d'occasion mais plus du double pour se procurer l'édition papier d'un grand quotidien du soir ? En matière d' « information » les sondés ne distinguent ni ne hiérar- 
chisent pas toujours leurs sources : famille, amis, collègues, voisins, medias traditionnels et nouveaux organes de presse principaux vecteurs de fake news. Quel sens donner au verbe s'informer alors que la défiance à l'encontre des professionnels de l'information et de toutes les catégories d'experts en général est à son comble ? Si un pays comme la Finlande obtient un indice de confiance envers les journalistes de $62 \%$, le Royaume-Uni $43 \%$, les USA $38 \%$, il est symptomatique qu'avec un score de seulement $30 \%$ la France se rapproche de celui qui ferme la marche et qui a sans aucun doute de bonnes raisons de douter de ses élites, la Grèce $(23 \%)^{52}$. Il est alors loisible d'établir une correspondance entre le niveau de défiance à l'égard des medias et le degré d'adhésion aux fake news et autres théories complotistes. Comme dans la loi de Gresham, la mauvaise monnaie chasse la bonne, la fausse information circule rapidement sur le marché tandis que l'information authentique est thésaurisée. Car si 17\% seulement des Français tirent principalement leurs informations des réseaux sociaux, ils sont $63 \%$ dans la tranche des 18-24 ans précisément la plus ouverte aux théories conspirationnistes et la plus imperméable à toute entreprise pédagogique de type « Les Décodeurs » ou « Désintox » car également la plus méfiante envers les institutions en général. Par définition, les responsables de ces rubriques de fact-checking ont pleinement conscience qu'ils ne peuvent que prêcher à des convertis. Un article du Monde ou de Libération documentant une fake news restera sans effet sur le public des sites identitaires comme fdesouche.com ou egaliteetreconciliation.fr. Lorsque l'on observe le contenu de ces sites dits de « ré-information » et plus généralement les forums de la « fachosphère $»^{53}$ en guerre contre le « mensonge média- tique », on peut toucher du doigt le discours de légitimation de ces acteurs opérant sur le marché de la post-vérité. En substance, ils nous disent qu'ils n'ont pas le choix. Comme pour les méthodes traditionnelles de propagande, ils ne savent pas si elles sont efficaces mais ils ne peuvent pas prendre le risque d'abandonner cette technique à leurs adversaires. «Nous sommes obligés de mentir pour pouvoir rester sur le marché des idées », plaide un étudiant en droit résidant en Nouvelle Aquitaine ${ }^{54}$. "Si les gens le croient c'est que c'est crédible », lui répond en écho un activiste identitaire ayant posté sur les réseaux sociaux des photos de voitures calcinées dans les rues de Bordeaux ou de Barbès prétendument brûlées par des supporters algériens. L'argument est d'autant plus imparable qu'en se plaçant sur le terrain de la conviction il échappe à celui de la démonstration logique donc de la réfutation. Ce phénomène des fake news atteste par ailleurs l'ambivalence de l'internet capable d'encourager l'expression de la démocratie participative et pétitionnaire (Change.org), «l'activisme du clic », tout autant que les formes d'extrémisme, de complotisme, de racisme et de populisme. Il met également à mal l'illusion communicationnelle consistant à croire que l'on démocratiserait l'information - donc la démocratie elle-même - en accroissant la masse des contenus et en multipliant le nombre et la vitesse de ce que l'on appelait au début des années 1990 «les autoroutes de l'information ». Les fake news en tant que nouvelle technique de propagande s'engouffrent d'autant plus facilement dans des sociétés gagnées par "l'infobésité »" ${ }^{55}$, la surcharge cognitive, l'excès d'informations, que le désir de savoir n'a pas remplacé le besoin de croire. Dans un monde toujours plus complexe et anxiogène, la propagande en général et les 
fake news en particulier ordonnent, simplifient et rassurent en nous désignant le camp du bien et celui du mal. Nous sommes tous complices et les intellectuels, en quête de sens et de vérité par vocation, ont seulement l'illusion d'échapper à ce mécanisme de cécité volontaire. Face à un choix, nous avons besoin de nous convaincre que nous prenons la bonne décision. Nous sommes complices du mécanisme qui nous trompe et le niveau d'instruction en accroissant notre curiosité intellectuelle et notre ouverture d'esprit peut nous rendre plus perméable aux idées extrêmes ou aberrantes. De ce point de vue, la sociologie cognitive confirme empiriquement les géniales intuitions de l'auteur de Propagandes ${ }^{56}$. Mais alors qu'il est question en France de légiférer sur les fausses nouvelles, donc de réglementer la vérité, le même Jacques Ellul nous alertait naguère sur le risque de réduction de la vérité aux faits. «Est-ce que l'homme est devenu homme en s'inclinant devant le Fait ? (...) La reconnaissance de la souveraineté du Fait, c'est l'élimination à plus ou moins longue déchéance de ce qui fait la grandeur, la spécificité, la vérité de l'homme ${ }^{57}$. » La Vérité est du domaine de la religion et de la morale. La science, elle, devrait se rappeler qu'il ne saurait exister de vérité que provisoire et relative.

\section{$R \cdot E \cdot F \cdot E \cdot R \cdot E \cdot N \cdot C \cdot E \cdot S$}

ALBERTINI D., DOUCET D., La Fachosphère. Comment l'extrême droite remporte la bataille $d u$ Net, Paris, Flammarion, 2016.

BARKUN M., «Les théories du complot comme connaissance stigmatisée », Diogène, 2015/1 (n 249-250), p. 168-176.

BERNAYS E., [1928], Propaganda. Comment manipuler l'opinion en démocratie, Préface de Normand Baillargeon, Paris, Zone/La Découverte, 2007

BRONNER G., La Démocratie des crédules, Paris, PUF, 2013

CARASCO A., La Croix, 2/2/2017, https://www. la-croix.com/Economie/Medias/Barometremedias-Francais-veulent-information-verifiee-2017-02-02-1200821914

CHASTENET P., The Conversation, « Nouvelle information ou vieille propagande ? ", 12/05/2017, http://theconversation.com/nouvelle-intox-ou-vieille-propagande-77447

DE LA FONTAINE J., Fables, « Le Loup et le Renard », t.2, Livre XI, III, Genève, Pierre L'Aîné, 1960.

DELANNOI G., GAUCHET M., HALEVI R., RAYNAUD Ph., «Sur la post-vérité », Le Débat, $\mathrm{n}^{\circ} 197$, novembre-décembre 2017.

DESORMEAUX D., GRONDEUX J., Le Complotisme : décrypter et agir, Canopé Éditions, 2017.

ELLUL J., [1967], Histoire de la propagande, coll. Que sais-je ?, Paris, Puf, 1976.

ELLUL J., Exégèse des nouveaux lieux communs, Paris, Calmann-Lévy, «Liberté de l'esprit », 1966.

ELLUL J., [1962], Paris, Propagandes, Econo- 
mica, 1990.

FRANKFURT H. G., On Bullshit, 1986, trad. fr. sous le titre De l'art de dire des conneries, Paris, 10/18, 2006.

HARSIN J., «Un guide critique des Fake News : de la comédie à la tragédie », Pouvoirs, 2018, $\mathrm{n}^{\circ} 164$.

JOUXA, « Du fact checking au fake checking », La revue européenne des médias et du numérique, $\mathrm{n}^{\circ}$ 44, automne 2017.

LOVELUCK B., " La démocratie au prisme du numérique », in TROUDE-CHASTENET P. (Dir.), Penser et panser la démocratie, Paris, Classiques Garnier, 2017.

MONIER N., Technikart, juin 2017.

NEWMAN N. et al., « Reuters Institute Digital News Report 2017 », https://reutersinstitute. politics.ox.ac.uk/sites/default/files/Digital\%20

News\%20Report\%202017\%20web_0.pdf

PARISER E., The Filter Bubble. What The Internet Is Hiding From You, New York, Penguin Press, 2011.

REBILLARD F., « La rumeur du PizzaGate durant la présidentielle de 2016 aux États-Unis. Les appuis documentaires du numérique et de l'Internet à l'agitation politique », Réseaux, vol. 202-203, n² 2017, p. 273-310.

ROBIN C., La Peur. Histoire d'une idée politique, Paris, Armand Colin, 2006.

SAUVAJOL-RIALLAND C., Infobésité : comprendre et maîtriser la déferlante d'informations, Vuibert, 2013

TAÏEB E., « La propagande revisitée », Quaderni, $\mathrm{n}^{\circ} 72,2010$, p. 5-18.

TROUDE-CHASTENET P., « Communication et société technicienne » in La Propagande, Cahiers Jacques-Ellul, n 4, Le Bouscat, L'Esprit du temps, Puf, 2006.
$\mathrm{N} \cdot \mathrm{O} \cdot \mathrm{T} \cdot \mathrm{E} \cdot \mathrm{S}$

\section{Sean Spicer.}

2. Revendiqué par Sean Spicer, alors porte-parole de la Maison-Blanche, lors d'une conférence de presse. «Je pense que parfois nous pouvons être en désaccord avec les faits ».

3. Élu mot de l'année 2017 par le dictionnaire Collins. 4. Ce dernier répondra ultérieurement aux trumpistes par un simple tweet : "La vérité vous rendra libres » $(\mathrm{Jn} 8,32)$ et en consacrant la prochaine Journée mondiale des communications sociales au phénomène des fake news.http://www.journee-mondiale.com/123/ journee-mondiale-des-communications-sociales.htm. p.1, consultée le 06/01/2017.

5. Hannah Ritchie, Special to CNBC.com, "Readall-about-it-the-biggest-fake-news-stories-of-2016", 30/12/2016.

6. Le Gorafi, 1/6/2016 (Le Figaro en verlan, fondé par les humoristes du groupe Jalons) : « Je ne suis pas méprisant... mais c'est plus fort que moi, quand je serre la main d'un pauvre, je me sens sale pour toute la journée ». Plusieurs salariés de Whirlpool l'avaient d'ailleurs interpellé à ce sujet lors de son arrivée sur le site de l'usine d'Amiens.

7.Sydney Schaedel, "Did the Pope Endorse Trump?", Fact.Check.org, 24/10/2016, et https://www.snopes. com/pope-francis-donald-trump-endorsement/ page consultée le 16/02/2018.

8 . Nous entendons par propagande la diffusion organisée d'informations - vraies ou fausses - destinées à faire - ou à ne pas faire - opérer à des acteurs ou à des groupes d'acteurs, à leur insu, une action (i.e. un vote) qu'ils n'auraient pas effectuée, ou pas de la même manière, sans cette information.

9. Jacques Ellul, [1967], Histoire de la propagande, PUF, 1976, p. 8-11. Cf. plus récemment, Emmanuel 
Taïeb, « La propagande revisitée », Quaderni, n 72, 2010, p. 5-18.

10.Patrick Chastenet, « Nouvelle information ou vieille propagande ?», The Conversation, 12/05/2017, http:// theconversation.com/nouvelle-intox-ou-vieille-propagande-77447, page consultée le 16/02/2018.

11. C'est globalement la thèse de Gil Delannoi, Marcel Gauchet, Ran Halévi, Philippe Raynaud, «Sur la post-vérité », Le Débat, n 197 , novembre-décembre 2017, p. 4-41.

12. Will Worley, "Donald Trump inspired Brexit campaign because facts don't work, says Leave founder Arron Banks", Independent, 30/06/2016. 'The remain campaign featured fact, fact, fact, fact, fact. It just doesn't work. You have got to connect with people emotionally. It's the Trump success".

13. Méthodes utilisées dès les primaires présidentielles du Parti républicain américain de 2016.

14. Le parti de Nikel Farage a délibérément placé l'immigration musulmane au centre de sa campagne mais il n'a pas été le seul Cf. Rajeev Syal, Peter Walker and Rowena Mason, « Boris Johnson tries to calm immigration row with call for amnesty », The Guardian, 16/06/2016.

15. Il existe un Bureau britannique des statistiques nationales qui porte le nom d'Office for National Statistics dont les travaux ont été utilisés pour démentir plusieurs fake news.

16. Cette expression fut d'abord utilisée en 2014 par Rob Shorthouse (Better Together), directeur de campagne des partisans du maintien de l'Écosse au sein du Royaume-Uni.

17. Rappelons que le grand théoricien anglais de la souveraineté étatique, Thomas Hobbes, auteur du Léviathan, disait de lui qu'il était né avec la peur et qu'il avait grandi avec elle. Sa mère avait accouché avant terme en apprenant les préparatifs du départ de «l'invincible Armada », quant à lui, il avouera que la peur avait été l'unique passion de sa vie. Cf. Corey Robin, La Peur. Histoire d'une idée politique, Paris, Armand Colin, 2006.

18. Jean de La Fontaine, Fables, « Le Loup et le Renard », t.2, Livre XI, III, Genève, Pierre L'Aîné, p. 161. 19. Jayson Harsin, «Un guide critique des Fake News: de la comédie à la tragédie », Pouvoirs, 2018, n 164, p. 99-119.

20. https://newrepublic.com/article/69929/no-exit, Michelle Cottle, No Exit, New Republic, 5 octobre 2009.; http://betsymccaughey.com/, page consultée le 16/02/22018.

21. https://www.facebook.com/notes/sarah-palin/ statement-on-the-current-health-care-debate/ 113851103434/ Page consultée le 16/02/22018.

22. Angie Drobnic Holan, "A look back at Lie of the Year, 2009 to 2016", 12/12/2017; http://www.politifact. com/truth-o-meter/article/2017/dec/12/look-back-lieyear-2009-2016/

23.Bob Cesca, "Debunking the Top 10 Most Egregious Republican Lies", Huffington Post, 25/03/2014 https:// www.alternet.org/tea-party-and-right/debunking-top10-most-egregious-republican-lies

24. https://www.youtube.com/watch?v= MU9V6eOFO38

25. Le bloggeur David Roberts, cité par Ran Halévi, utilisait déjà ce concept en 2010 mais seulement pour dénoncer les excès de la polarisation partisane aux États-Unis au détriment de l'intérêt général.

26. Lauren Caroll, Linda Qiu, "Looking back at Lie of the Year", 13/12/2016, http://www.politifact.com/ truth-o-meter/article/2016/dec/13/looking-back-lieyear/

27. "Fake news is made-up stuff, masterfully manipulated to look like credible journalistic reports that are easily spread online to large audiences willing to believe the fictions and spread the word."

28. Alexandre Joux, « Du fact checking au fake 
checking ", La revue européenne des médias et du numérique, $\mathrm{n}^{\circ} 44$, automne 2017, page consultée le 16/02/2018.

29. Franck Rebillard, «La rumeur du PizzaGate durant la présidentielle de 2016 aux États-Unis. Les appuis documentaires du numérique et de l'Internet à l'agitation politique », Réseaux, vol. 202-203, n 2 , 2017, p. 273-310.

30. "Trump Rally Chant", https://www.snopes.com/ trump-rally-chant/. Page consultée le 16/02/2018.

31.Nick Newman et al., « Reuters Institute Digital News Report $2017 »$, p. 11 et p. 102, https://reutersinstitute.politics.ox.ac.uk/sites/default/files/Digital $\% 20$ News\%20Report\%202017\%20web_0.pdf.

32. Institut Médiamétrie, Étude réalisée du 27 janvier au 16 février 2016 auprès de 3105 individus de 18 ans et plus par téléphone ou en ligne. L'étude dirigée par Nick Newman donne le chiffre de $40 \%$ sans toutefois indiquer s'il s'agit de la source privilégiée d'information.

33. « Dans la capitale mondiale des fausses informations », http://www.slate.fr/story/137795/capitalemondiale-fausses-informations, page consultée le 16/02/2018.

34.Benoit Le Corre, « En Macédoine, Trump est une machine à cash pour des sites d'info crapuleux », $L^{\prime} O b s$ avec Rue 89, 4/11/2016.

35. Cette notion est entérinée par le droit américain à travers un avis célèbre prononcé par le juge Oliver Wendel Holmes, contre une décision de la Cour suprême américaine condamnant des militants pour avoir distribué des tracts prosoviétiques pendant la guerre - Abrams vs United States, 250 U.S. 616 (1919).

36. Benjamin Loveluck, «La démocratie au prisme du numérique », in Patrick Troude-Chastenet (Dir.), Penser et panser la démocratie, Classiques Garnier, 2017, p. 150.

37. Jayson Harsin propose une adaptation de la maxime de Metternich : «Quand la France éternue, toute l'Europe s'enrhume ». « Ce sont les États-Unis aujourd'hui qui éternuent (à vous de décider qui s'enrhume) ». Op. cité. p. 99.

38.David Leonhardt, Stuart A. Thompson, “Trump'Lies”, The New York Times, 14/12/2017.

39. Je remercie Emmanuel Taïeb d'avoir attiré mon attention sur l'article de Michael Barkun, « Les théories du complot comme connaissance stigmatisée », Diogène, 2015/1 (n² 249-250), p. 168-176.

40. Jacques Ellul, [1962], Propagandes, Economica, 1990, Patrick Troude-Chastenet, "Communication et société technicienne" in La Propagande, Cahiers Jacques-Ellul, ${ }^{\circ}$ 4, L’Esprit du temps, 2006, p. 129-145.

41. Ran Halévi, op.cit. p. 36.

42. Edward Bernays, [1928], Propaganda. Comment manipuler l'opinion en démocratie, Zone/La Découverte, 2007, p. 104.

43. On peut traduire approximativement bullshit par foutaises, conneries, baratin.

44. Eric Ambler, A dirty story, 'Never tell a lie when you can bullshit your way through', cité in Harry G. Frankfurt, On Bullshit, 1986. Traduit sous le titre De l'art de dire des conneries, 10/18, 2006.

45. Ran Halévi, op. cit., p. 35. À contre-courant, certains pointent la responsabilité de la gauche, et même des universitaires, dans l'avènement de la post-vérité, car ils ont considéré que les faits étaient « inaccessibles » (question de point de vue ou d'interprétation) et que la « vérité » n'existait pas. Andrew Calcutt, «Comment la gauche libérale a inventé la post-vérité » ?, The Conversation, 6 décembre 2016, https://theconversation.com/comment-la-gaucheliberale-a-invente-la-post-verite-69310, Consultée le $16 / 02 / 2018$.

46. Les activistes ayant utilisé ce forum s'attribuent la victoire de Trump: 'We actually elected a meme as 
president': How 4chan celebrated Trump's victory, Washington Post, 9 novembre 2016.

47. Eli Pariser, The Filter Bubble. What The Internet Is Hiding From You, New York, Penguin Press, 2011. 48. Jayson Harsin, op.cit. p. 108, note 22, définit les bots comme des "programmes robotisés qui sont capables de discuter avec leurs utilisateurs et de leur proposer des services selon leurs demandes ».

49. A.A. Casilli, «Qui a fait élire Trump ? Pas les algorithmes, mais des millions de «tâcherons du clic»" sous-payés », Casilli.fr, 17 novembre 2016.

50. Jayson Harsin, op.cit.

51. Benjamin Loveluck, « La démocratie au prisme du numérique », in Patrick Troude-Chastenet (Dir.), Penser et panser la démocratie, Classiques Garnier, 2017, p. 150.

52. Nick Newman et al., « Reuters Institute Digital News Report 2017 », 76\% des Français pensent que les journalistes ne sont pas indépendants, Baromètre La Croix, 2/2/2017.

53. Dominique Albertini, David Doucet, La Fachosphère. Comment l'extrême droite remporte la bataille du Net, Paris, Flammarion, 2016.

54. Nicolas Monier, Technikart, juin 2017, p. 69.

55. Caroline Sauvajol-Rialland, Infobésité : comprendre et maîtriser la déferlante d'informations, Vuibert, 2013.

56. Jacques Ellul, [1962], Propagandes, Economica, 1990, et Gérald Bronner, La Démocratie des crédules, Paris, PUF, 2013 qui signale notamment que $51 \%$ de la population new-yorkaise, pourtant plus instruite que la moyenne nationale, était persuadée que le gouvernement avait été prévenu de l'imminence des attentats et n'avait rien fait pour les déjouer.

57. Jacques Ellul, Exégèse des nouveaux lieux communs, Calmann-Lévy, « Liberté de l'esprit », 1966, p. 203.

\section{$R \cdot E ́ \cdot S \cdot U \cdot M \cdot E ́$}

Le phénomène des fake news a commencé a polarisé l'attention des observateurs en 2016 dans le cadre de la campagne référendaire au Royaume-Uni portant sur le Brexit et lors de l'élection présidentielle aux États-Unis. La combinaison d'informations trompeuses et de prétendus faits alternatifs nous a-t-elle fait basculer imperceptiblement dans une nouvelle ère de la communication connue sous le nom de post-vérité ? Si cette situation n'est pas totalement inédite et rappelle opportunément que la propagande ne se conjugue ni au passé, ni exclusivement au-delà de nos frontières elle comporte sa part de spécificité. Elle atteste de l'ambivalence de l'internet capable d'encourager l'expression de la démocratie participative tout autant que les formes d'extrémisme, de complotisme et de populisme. Elle ruine également l'illusion communicationnelle consistant à croire que l'on démocratiserait l'information - donc la démocratie elle-même - en accroissant la masse des contenus et en multipliant le nombre et la vitesse de ce que l'on appelait naguère « les autoroutes de l'information».

\begin{abstract}
The phenomenon of fake news began to draw observers' attention in 2016, during the UK referendum campaign on Brexit and the US presidential election. Has the combination of misleading information and so-called alternative facts tipped us imperceptibly into a new, "post-truth" era of communication? Even if this phenomenon is not totally unprecedented, and serves to remind us that propaganda exists not only in the past or in other countries, it has its own unique aspects. It demonstrates the internet's ambiguity in encouraging the expression not only of participatory democracy but also of extremism, populism, and conspiricism. It also
\end{abstract}


destroys the illusion that if we increase the volume of information, and the speed at which it is delivered on what was formerly called "the information superhighway," we will democratize information - and therefore democracy itself. 
